\title{
Inter-subject Connectivity-Based Parcellation of a Patch of Cerebral Cortex
}

\author{
Pauline Roca ${ }^{1,2}$, Alan Tucholka ${ }^{1,2,3}$, Denis Rivière ${ }^{1,2}$, Pamela Guevara ${ }^{1,2}$, \\ Cyril Poupon ${ }^{1,2}$, and Jean-François Mangin ${ }^{1,2}$ \\ 1 CEA Saclay, Neurospin/LNAO, Bât 145, 91191 Gif-sur-Yvette cedex, France \\ 2 Institut Fédératif de Recherche 49, Gif-sur-Yvette, France \\ ${ }^{3}$ INRIA Saclay, Parietal, France
}

\begin{abstract}
This paper presents a connectivity-based parcellation of the human post-central gyrus, at the level of the group of subjects. The dimension of the clustering problem is reduced using a set of cortical regions of interest determined at the inter-subject level using a surfacebased coordinate system, and representing the regions with a strong connection to the post-central gyrus. This process allows a clustering based on criteria which are more reproducible across subjects than in an intra-subject approach. We obtained parcels relatively stable in localisation across subjects as well as homogenous and well-separated to each other in terms of connectivity profiles. To address the parcellation at the inter-subject level provides a direct matching between parcels across subjects. In addition, this method allows the identification of subject-specific parcels. This property could be useful for the study of pathologies.
\end{abstract}

Keywords: human connectome, anatomical connectivity, cortical parcellation.

\section{Introduction}

In-vivo parcellation of the human cortex into functional brain areas is a major goal in neurosciences and clinical surgery. Anatomical connectivity based on diffusion-weighted imaging has been used to address this problem, based on the hypothesis that functional regions have a specific connectional fingerprint [1. It presents the advantage of providing optimal basic elements for the construction of the human connectome [2]. These elements are defined at a scale maximizing connectivity-based similarities across subjects. However the huge dimension of connectivity data leads to important difficulties. A first approach consists in performing clustering of a small area of the brain using cross-correlation between connectivity profiles towards the full brain [3. However, the huge dimension of the data has prevented from performing the clustering at inter-subjects level as well as on the whole brain cortex.

To overcome some of the limitations, a key idea is to collapse the connectivity profiles using a set of Regions Of Interest: connectivity weights are summed up across each ROI. Some approaches use a priori anatomical information such as

T. Jiang et al. (Eds.): MICCAI 2010, Part II, LNCS 6362, pp. 347-354 2010.

(C) Springer-Verlag Berlin Heidelberg 2010 
lobar or gyri cortex segmentation [45] or regions of interest from invasive tracing primate studies [6]. When there is a correspondence across subjects between these segmentations, it provides a direct way to perform the clustering at the level of the group of subjects 445. The robustness is largely increased because the clustering is focusing on profiles reproducible across subjects. Nevertheless using a cortex parcellation to infer another presents an evident bias. Another approach [7] avoids this by infering automatically the required segmentation from the connectivity data, independently for each subject. However this method has shown poor reproducibility across subjects.

The main contribution of this paper is to push this last approach at the level of the group of subjects. For this purpose, the set of ROIs obtained from the connectivity data is defined directly at the group level. This is achieved using a 2D coordinate system providing correspondence between the cortical surfaces across subjects [8]. Once the profiles have been collapsed using this set of ROIs, they are clustered following two different stategies. The profiles are either gathered alltogether or averaged across subjects. In the following the whole method is applied to parcellate the right post-central gyrus of a group of ten subjects.

\section{Material and Methods}

\subsection{Data and Pre-processings}

The present study has been performed on $N=10$ subjects of the NMR database 9. Diffusion weighted data were acquired with HARDI scheme, resulting in high-quality datasets based on 200 directions of diffusion and a $b$ value of 3000 $\mathrm{s} / \mathrm{mm}^{2}$. Furthermore, the alignment between diffusion data and T1-weighted images is achieve by a dedicated MR sequence and distortion corrections. In order to recover the cortico-cortical anatomical connectivity, a streamline probabilistic tractography [10] is performed in the white matter volume using a field of Orientation Distribution Functions (ODF) calculated from the DW data with the analytical QBall model described in [11.

The grey/white matter interface is reconstructed using Freesurfer [12]. We created a slightly inflated inter-subject average mesh for visualisation purpose [8]. A point-to-point correspondence between all the meshes provides a way to analyze the connectivity data in a common space. The post-central gyrus is determined from the automatic surface labeling process of Freesurfer, which parcellates the surface into 66 anatomical cortical regions [13].

The cortical connectivity matrix of the post-central gyrus of each subject is calculated using the previous tractography results and surfacic data [7. For each subject we obtained a matrix of size $(G, C)$ where $C$ and $G$ are the number of vertices of the cortex mesh and of the post-central gyrus respectively. Each line of these matrices corresponds to the connectivity profile from one post-central gyrus vertex of one subject towards the full cortex defined in Freesurfer coordinate system. In order to take into account the uncertainty of the tractography, 
a Gaussian smoothing of the connectivity matrix was applied on the surface $(\sigma=5 \mathrm{~mm})$.

\subsection{Inter-subject Dimension Reduction of the Connectivity Data}

In this part the objective is to compress the connectivity profiles in a way maximizing similarities across subjects. The sources of variability of connectivity profiles across subjects are multiple:

1. the artifactual variability created by weaknesses of the diffusion-based tracking scheme;

2. the non perfect spatial normalization;

3. the purely anatomical variability acting on areas of the parcels and on density and repartition of the fibers inside each parcel.

Geodesic smoothing of connectivity information is a first way to overcome the resulting difficulties. A second complementary way consists in performing a kind of group analysis detecting the cortical regions with reproducible connectivity to the input patch (here post-central gyrus). For each subject the post-central gyrus connectivity profile is computed, that is to say the average of the connectivity profiles across gyrus vertices (Fig. 1). This gyrus profile is normalized by the number of tracts reaching the gyrus. Then an average of these profiles across subjects is performed. The complete surface is masked to keep only nodes that received at least ten fiber tracts from at least half of the subjects.

The resulting average connectivity profile is then mapped on the average mesh(Fig. 1), represented as a texture. A watershed is computed for the average connectivity profile texture in order to split the cortical surface into catchment basins [7. Each basin catches a set of tracts supposed to connect the postcentral gyrus with a specific brain area (Fig. 1). This idea justifies the use of this set of basins to collapse the profiles in an optimal way regarding connectivity. Indeed, because of the inter-subject variability, the regions with a strong connection to the input patch (appearing in the connectivity profile of each

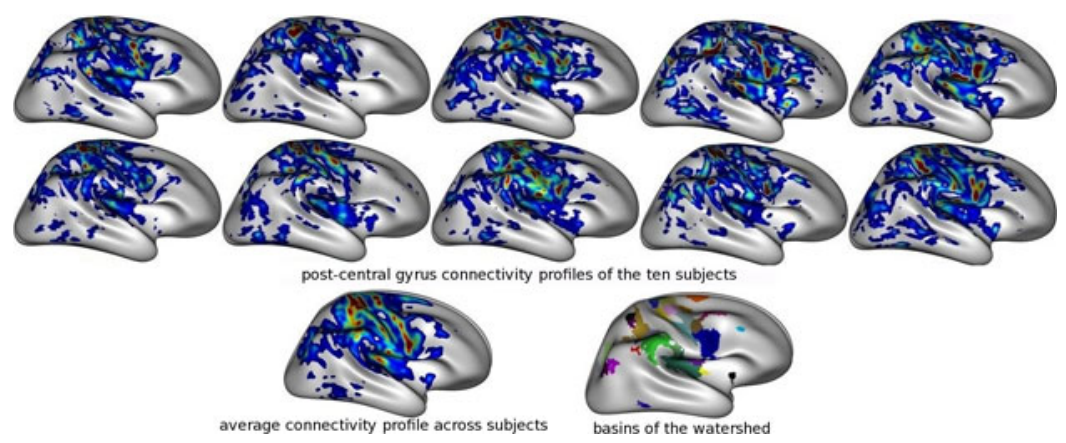

Fig. 1. Different connectivity profiles and associated watershed basins 

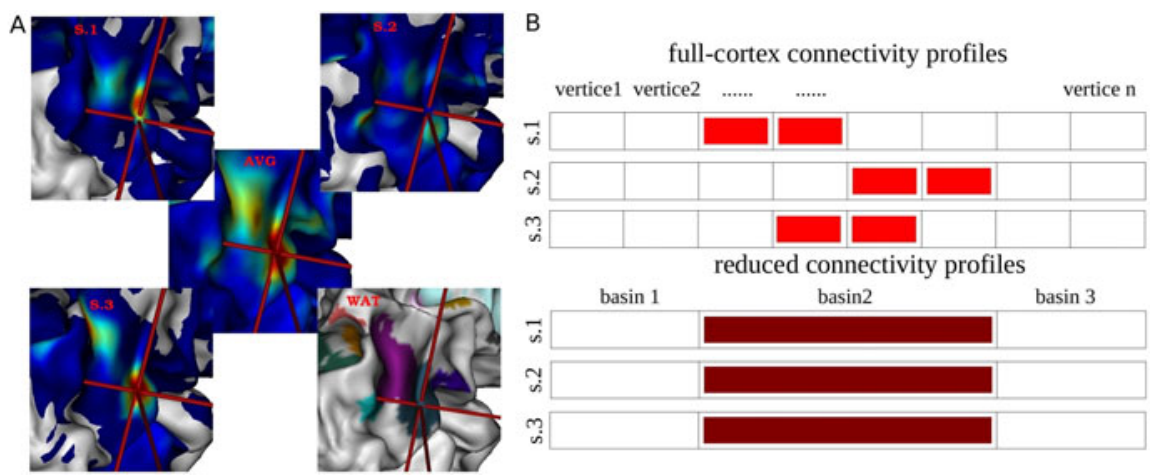

Fig. 2. Contribution of the watershed. A. s1,s2,s3: Zoom on one part of the post-central gyrus connectivity profile for three subjects; avg. Averaged connectivity profile; wat. Associated watershed basins. B. The same illustration in 1D.

subject) do not match perfectly when they are mapped onto the average cortex mesh(Fig,2,A.s1.s2.s3). When these profiles are averaged across subjects, the previous regions are grouped together to form a unique region (Fig, 2 A.avg). The watershed algorithm can then isolate this region as a basin (Fig, 2 A.wat). Note that a more sophisticated fMRI-inspired group analysis could be performed.

To conclude this step, these basins are used to reduce the dimension of the connectivity profiles: for each subject independently, all the vertices connectivity profiles ( $G$ profiles of size $C$ ) are collapsed to get $G$ connectivity profiles of dimension $n_{\text {basins }}$ (number of basins). This dimension reduction cancels out most of the inter-individual variability mentioned above. The connectivity information resulting from each brain area represented by a catchment basin is summarized to one single weight (Fig, 2 B).

\subsection{Cortical Parcellation}

The reduced connectivity profiles of all the subjects are normalized using $L_{2}$ norm and then clustered using two alternative strategies.

First, the post-central gyrus vertices connectivity profiles of all the subjects (Fig, 3. A) are concatenated into a big matrix of size $\left(N * G, n_{\text {basins }}\right)$ (Fig 3, B). Hence the clustering algorithm deals with all the profiles without knowledge of the underlying subjects. The clustering is performed with the classical kmedoids algorithm (called PAM in R language [14) and the Euclidean distance between profiles as dissimilarity measure. The number of clusters superior to two and maximizing the average silhouette width of the clustering is chosen. A cluster usually includes profiles stemming from most of the subjects. Therefore, the end result is one specific parcellation for each subject, but with a direct matching between the parcels across the subjects.

In the second approach, the same connectivity profiles are averaged across subjects for each vertice of the post-central gyrus (Fig 3, C). Then they are 


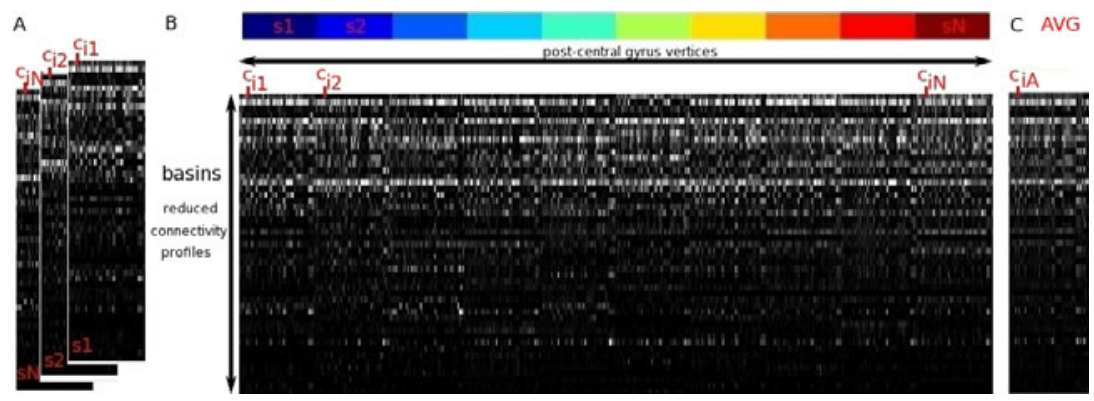

Fig. 3. Connectivity profiles of gyrus vertices: A. By subject; B. Concatenated for all the subjects; C. Averaged across subjects $\left(c_{i A}=\frac{1}{N} \sum_{s=1}^{N} c_{i s}\right)$

clustered using the same algorithm (PAM). By doing this, one single parcellation is obtained from a connectivity matrix of relatively small size $\left(G, n_{\text {basins }}\right)$.

The first approach has the advantage to preserve the inter-subject variability. Indeed the surface-based coordinate system is just used to define the input patch and to regroup the vertices into watershed basins for dimension reduction. The information related to the node localization in the coordinate system is not used by the clustering. When averaging, individual information is canceled out to provide a group result.

\section{Results}

The results are quite encouraging. The parcellations obtained by the two previous approaches are consistent with each other. For the average subject clustering, the optimal number of clusters is five (Fig 4) while with the concatenation approach, the optimal number is seven (Fig 5 ). For the concatenation approach, parcels have their own connectivity pattern, which is quite similar across subjects and relatively well-separated from other parcels (Fig, 5 , B). Note that in
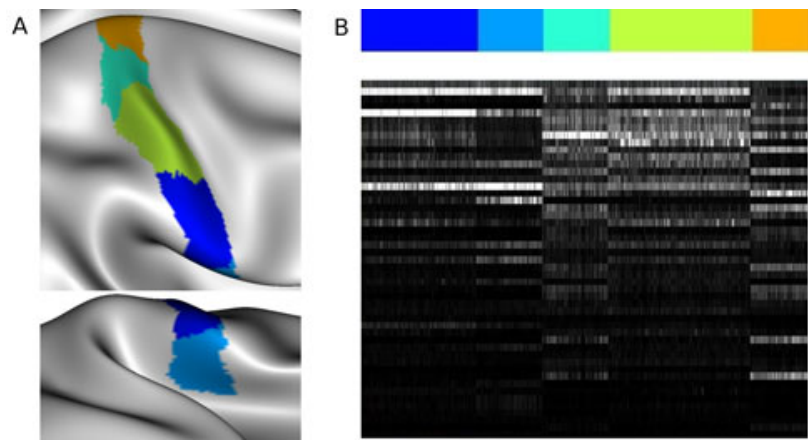

Fig. 4. Average approach: A. Parcellation; B. Averaged connectivity profiles (like in Fig 3 C) ordered by parcel 


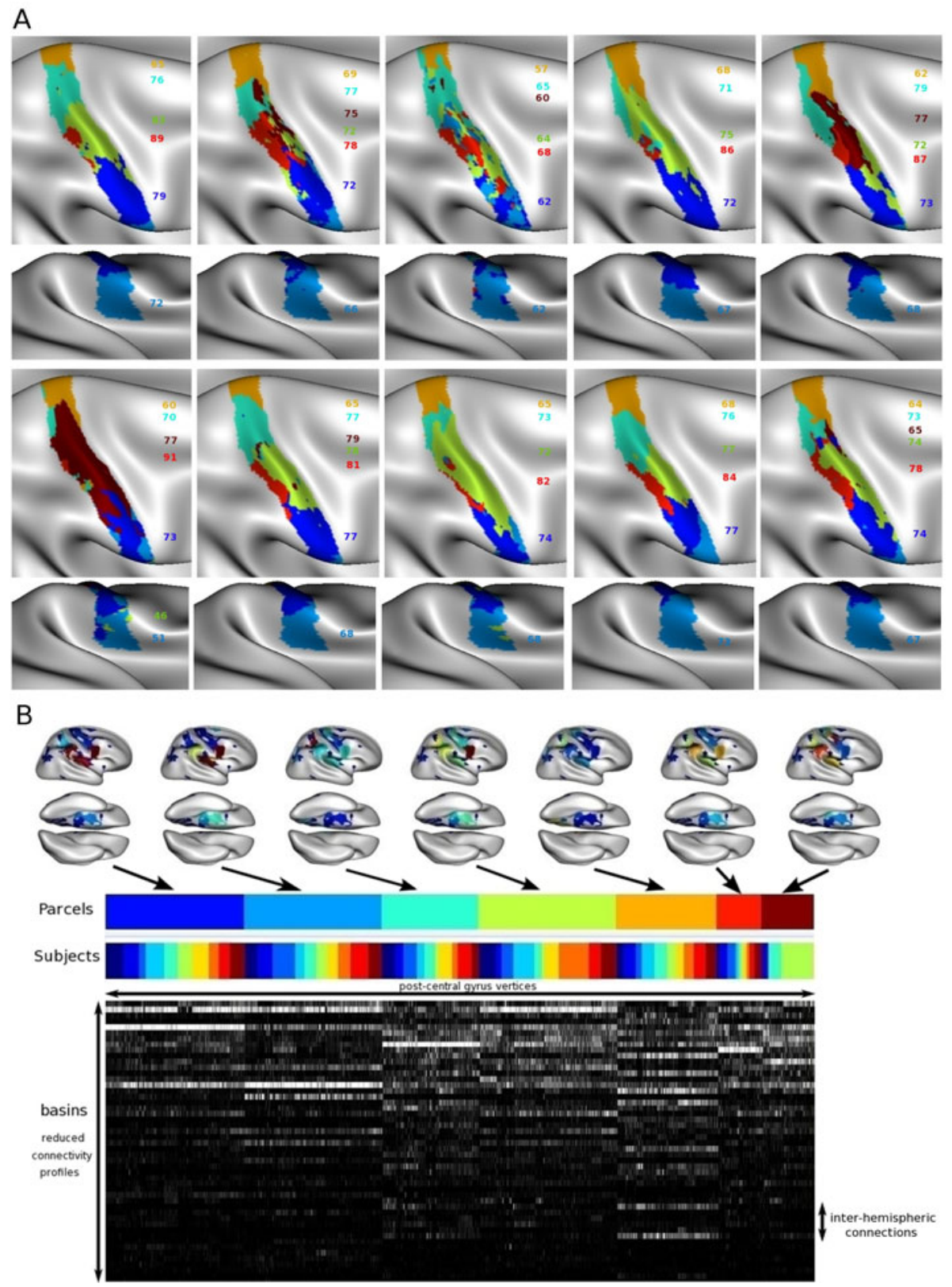

Fig. 5. Concatenation Approach: A. Parcellation for the ten subjects with the amount of tracts used by the clustering, by subject and parcel in percentage; B. i.Connectivity profiles of each parcel averaged across subjects and mapped on the inflated average mesh; ii. Concatenated connectivity profiles (like Fig 3 B) ordered by parcel 
the concatenation approach, while the global number of parcels is seven, some parcels exist only for a subset of subjects. With a set of subjects composed of distinct sub-groups (patients vs controls for example) this flexibility could allow the identification of group-specific parcels.

The correspondence between the parcels found with the two approaches is straightforward considering the connectivity profiles and the localization (cf. parcels colors matching between Fig 4 and 5). One of the additional clusters for the concatenation approach stems mainly from two subjects (claret color in 5). It could result from artifacts in the underlying diffusion data leading to a spurious cluster. The second additional cluster (red) results from a split of the green cluster into two different parcels. This subdivision can not be detected by the average approach because of the interindividual variability of the localization of the boundary between green and red parcels. Except the claret parcel, the results of the concatenation approach are quite reproducible across subjects in terms of connectivity profiles and localization on the cortex. Note that the topology of the set of parcels is consistent across subjects and corresponds to the topology obtained by the average approach. The shapes and areas of each parcel and the localization of the boundaries between parcels, however, vary largely between subjects, which is consistent with literature on architectony.

\section{Discussion}

In this paper, we have shown that addressing the connectivity-based parcellation problem at an inter-subject level can lead to reproducible results even without using a priori knowledge for dimension reduction. In the optimal case, the watershed onto the average connectivity profile across subjects allows to regroup the subject-specific connections into one ROI used later to reduce the connectivity dimension. When it is not the case, the application of fMRI-inspired group analysis could provide better results and deserves our attention in the future. When dealing with a very large set of brains and targetting the parcellation of the complete cortical surface, the average approach may be the only reasonable way because of computational considerations. Note that extending the parcellation algorithm to the complete surface can be done iteratively like in [7]. On the other hand, when the concatenation approach is affordable, it provides a very attractive tool. Indeed, the connectome nodes are defined at the level of each subject, providing a connectivity-based referential to be used for various applications. For instance this parcellation could be the basis for fMRI or cortical thickness studies. The connectivity-based parcellation, indeed, provides an architectural spatial normalization of greater value than the traditional normalization based on the cortical surface geometry. Adapting the connectome to each subject connectivity data is also of great interest to push further the study of its topology using graph theory [15. Defining the connectome nodes from the standard geometry based normalization indeed, is bound to hide the fine scale structure of the brain connectivity. In addition, the concatenation approach allows the identification of group-specific parcels and could be useful for the study of pathologies. 


\section{References}

1. Passingham, R.E., Stephan, K.E., Kötter, R.: The anatomical basis of functional localization in the cortex. Nature 3, 606-616 (2002)

2. Sporns, O., Tononi, G., Kötter, R.: The human connectome: A structural description of the human brain. PLOS Computational Biology 1, 245-251 (2005)

3. Anwander, A., Tittgemeyer, M., von Cramon, D., et al.: Connectivity-based parcellation of brocas area. Cerebral Cortex 17, 816-825 (2007)

4. Guevara, P., Perrin, M., Cathier, P., et al.: Connectivity-based parcellation of the cortical surface using Q-ball imaging. In: 5th Proc. IEEE ISBI, Paris, France, pp. 903-906 (2008)

5. Jbabdi, S., Woolrich, M.W., Behrens, T.E.J.: Multiple-subjects connectivity-based parcellation using hierarchical dirichlet process mixture models. NeuroImage 44 , 373-384 (2009)

6. Rushworth, M.F.S., Behrens, T.E.J., Johansen-Berg, H.: Connections patterns distinguish 3 regions of human parietal cortex. Cerebral Cortex 16, 1418-1430 (2005)

7. Roca, P., Riviere, D., Guevara, P.: Tractography-based parcellation of the cortex using a spatially-informed dimension reduction of the connectivity matrix. In: Yang, G.-Z., Hawkes, D., Rueckert, D., Noble, A., Taylor, C. (eds.) MICCAI 2009. LNCS, vol. 5761, pp. 935-942. Springer, Heidelberg (2009)

8. Fischl, B., Sereno, M., Tootell, R., et al.: High-resolution intersubject averaging and a coordinate system for the cortical surface. Human Brain Mapping 8, 272 284 (1999)

9. Poupon, C., Poupon, F., Allirol, L., et al.: A database dedicated to anatomofunctional study of human brain connectivity. In: HBM 2006 (2006)

10. Perrin, M., Cointepas, Y., Cachia, A., Poupon, C.: Connectivity-based parcellation of the cortical mantle using q-ball diffusion imaging. Int. J. Biomed. Imaging (2008)

11. Descoteaux, M., Angelino, E., Fitzgibbons, S., et al.: Regularized, fast, and robust analytical q-ball imaging. Magnetic Resonance in Medicine 58, 497-510 (2007)

12. Dale, A.M., Fischl, B., Serenot, M.I.: Cortical surface-based analysis. i. segmentation and surface reconstruction. NeuroImage 9, 179-194 (1999)

13. Desikan, R., Ségonne, F., Fischl, B., et al.: An automated labeling system for subdividing the human cerebral cortex on mri scans into gyral based regions of interest. Neuroimage 31, 968-980 (2006)

14. Kaufmann, L., Rousseeuw, P.J.: Finding groups in data: an introduction to cluster analysis. Wiley Interscience, Hoboken (1990)

15. Hagmann, P., Cammoun, L., Gigandet, X., et al.: Mapping the structural core of human cerebral cortex. PLOS Computational Biology 6(7), 1479-1493 (2008) 